\title{
Appendices for Variational Bayes for Functional Data Registration, Smoothing, and Prediction
}

\author{
Cecilia Earls*, Giles Hooker*
}

\section{Appendix A}

Below, in detail, are the specifications for the hierarchical Bayesian registration model discussed in this paper. The first section includes the basic model for functional data registration also found in Section 2. Section A.2 describes the MCMC sampling scheme for this model.

\section{A.1 Functional Registration}

As discussed in Section 2, the initial assumption of this model is that we are interested in registering functional data, $X_{i}(t), i=1, \ldots, N$, where these data are either observed directly over a set of time points, $\mathbf{t}=\left(t_{1} \ldots t_{p}\right)^{\prime}$, or are estimated from noisy observations, $\mathbf{Y}_{i}=\left(Y_{i}\left(t_{1}\right), \ldots, Y_{i}\left(t_{p}\right)\right)^{\prime}$. We assume a Gaussian noise process such that for each observation $\mathbf{Y}_{i}$,

$$
f\left(Y_{i}\left(t_{j}\right) \mid X_{i}\left(t_{j}\right), \sigma^{2}\right)=N\left(X_{i}\left(t_{j}\right), \sigma^{2}\right) \text { for } i=1, \ldots, N, \quad j=1, \ldots, p,
$$

which results in the joint distribution of all observations,

$$
f\left(\mathbf{Y} \mid \mathbf{X}, \sigma^{2}\right)=\prod_{i=1}^{N} N_{p}\left(\mathbf{X}_{i}, \sigma^{2} I\right),
$$

where $\mathbf{Y}$ is the matrix such that the observation for function $X_{i}(t)$ at time point $t_{j}$ is in the $i$ th row and the $j$ th column, $\mathbf{X}$ is the matrix of the corresponding means for each entry in $\mathbf{Y}$, and $\mathbf{X}_{i}=\left(X_{i}\left(t_{1}\right), \ldots, X_{i}\left(t_{p}\right)\right)^{\prime}$, the vector of evaluations of the functions $X_{i}(t)$ at time points $\mathbf{t}=\left(t_{1}, \ldots, t_{p}\right)^{\prime}$.

When the observations are observed noisily, the registered functions and noise variance are characterized by the following prior distributions:

$$
\mathbf{X}_{i}\left(\mathbf{h}_{i}\right) \mid z_{0 i}, z_{1 i}, \mathbf{f}, \gamma_{R}, \lambda_{X} \sim N_{p}\left(z_{0 i} \mathbf{1}+z_{1 i} \mathbf{f}, \gamma_{R}^{-1} \boldsymbol{\Sigma}+\boldsymbol{\Sigma}_{X}\right), \quad i=1 \ldots N
$$

\footnotetext{
*Department of Biological Statistics and Computational Biology, 1198 Comstock Hall, Ithaca, NY
} 14853 


$$
\begin{aligned}
\boldsymbol{\Sigma}_{X} & =\eta_{X}^{-1} \mathbf{P}_{1}+\lambda_{X}^{-1} \mathbf{P}_{2}, \text { and } \\
\sigma_{y}^{2} & \sim I G(a, b) .
\end{aligned}
$$

However, If each function $X_{i}(t)$ is observed directly over $\mathbf{t},(1)$ assumes the roll of the distribution of the observed data and the covariance matrix, $\boldsymbol{\Sigma}_{X}$, designed to penalize roughness in the unregistered functions is excluded. This results in the following data distribution,

$$
\mathbf{X}_{i}\left(\mathbf{h}_{i}\right) \mid z_{0 i}, z_{1 i}, \mathbf{f}, \gamma_{R}, \lambda_{X} \sim N_{p}\left(z_{0 i} \mathbf{1}+z_{1 i} \mathbf{f}, \gamma_{R}^{-1} \boldsymbol{\Sigma}\right), \quad i=1 \ldots N
$$

In both scenarios, we assume the following additional priors:

$$
\begin{aligned}
\eta_{X} & \sim G(c, d) \\
\lambda_{X} & \sim G(c, d) \\
\mathbf{h}_{i}\left(t_{j}\right) & =t_{1}+\sum_{k=2}^{j}\left(t_{k}-t_{k-1}\right) \exp \left(w_{i}\left(t_{k}\right)\right), \quad i=1 \ldots N, \quad j=1 \ldots p, \\
\mathbf{w}_{i} \mid \gamma_{w} & \propto N_{p-1}\left(\mathbf{0}, \gamma_{w}^{-1} \mathbf{\Sigma}+\lambda_{w}^{-1} \mathbf{P}_{2}\right) \mathbf{1}\left\{t_{1}+\sum_{k=2}^{p}\left(t_{k}-t_{k-1}\right) \exp \left(w_{i}\left(t_{k}\right)\right)=t_{p}\right\}, \quad i=1 \ldots N, \quad s, t \in \mathcal{T}, \quad z_{0 N}=-\sum_{i=1} z_{0 i}, \\
z_{0 i} \mid \sigma_{z 0}^{2} & \sim N\left(0, \sigma_{z 0}^{2}\right) \quad i=1 \ldots(N-1), \\
\sigma_{z 0}^{2} & \sim I G(a, b), \\
z_{1 i} \mid \sigma_{z 1}^{2} & \sim N\left(1, \sigma_{z 1}^{2}\right), \quad i=1 \ldots N, \\
\sigma_{z 1}^{2} & \sim I G(a, b), \\
\mathbf{f} \mid \eta_{f}, \lambda_{f} & \sim N_{p}\left(0, \boldsymbol{\Sigma}_{f}\right), \\
\mathbf{\Sigma}_{f} & =\eta_{f}^{-1} \mathbf{P}_{1}+\lambda_{f}^{-1} \mathbf{P}_{2}, \\
\eta_{f} & \sim G(c, d), \text { and } \\
\lambda_{f} & \sim G(c, d),
\end{aligned}
$$

where $\mathrm{a}, \mathrm{b}, \mathrm{c}$, and d are fixed hyperparameters.

$\boldsymbol{\Sigma}$ is a fixed matrix designed to penalize variation in any direction from the corresponding mean of the distribution in which it is utilized. It is composed of two matrices, $\mathbf{P}_{1}$ and $\mathbf{P}_{2}$, such that $\boldsymbol{\Sigma}=\mathbf{P}_{1}+\mathbf{P}_{2} . \mathbf{P}_{1}$ penalizes variation from the mean in constant and linear directions, and $\mathbf{P}_{2}$ penalizes variation from the mean in directions of curvature. For the distribution on the registered functions, $\boldsymbol{\Sigma}$ penalizes variation from a vertical shift and scaling of the target function. In the distribution of the base functions, $\boldsymbol{\Sigma}$ penalizes variation from the identity warping. The fixed parameters $\gamma_{R}$ and $\gamma_{w}$ determine the degree of these penalties for the registered functions and the base functions, respectively. 
$\mathbf{P}_{2}$ is also used to penalize curvature in the registered functions, base functions, and the target function with associated smoothing parameters $\lambda_{X}, \lambda_{w}$, and $\lambda_{f}$. Further details of the construction of $\mathbf{P}_{1}$ and $\mathbf{P}_{2}$ are found in Earls and Hooker (2014). Also note, in the prior on the base functions (3), we are allowing $\mathbf{P}_{2}$ to be more generally interpreted as a penalty on severe deformations of the unregistered functions. Here, $\mathbf{P}_{2}$ may be either a penalty on the squared second derivative of the base functions (as above) or a penalty on the squared first derivative of the base functions.

\section{A.2 MCMC Sampling}

Using these assumptions, the following full conditional distributions are derived to run a MCMC sampler:

$$
\begin{aligned}
& \mathbf{X}_{i} \mid \text { rest } \sim N_{p}\left(\left(\sigma_{y}^{-2} \mathbf{I}_{p}+\boldsymbol{\Sigma}_{X}^{-1}\right)^{-1}\left(\sigma_{y}^{-2} \mathbf{I}_{p} \mathbf{Y}_{i}+\boldsymbol{\Sigma}_{X}^{-1}\left(z_{0 i} \mathbf{1}_{p}+z_{1 i} \mathbf{f}\left(\mathbf{h}_{\mathbf{i}}^{-\mathbf{1}}\right)\right),\left(\sigma_{y}^{-2} \mathbf{I}_{p}+\boldsymbol{\Sigma}_{X}^{-1}\right)^{-1}\right),\right. \\
& \mathbf{f} \mid \text { rest } \sim N_{p}\left(\boldsymbol{\mu}_{\mathbf{f} \mid \text { rest }}, \boldsymbol{\Sigma}_{\mathbf{f} \mid \text { rest }}\right) \text {, } \\
& \boldsymbol{\Sigma}_{\mathbf{f} \mid \text { rest }}=\left(\sum_{i=1}^{N} z_{1 i}^{2}\left(\gamma_{R}^{-1} \boldsymbol{\Sigma}+\boldsymbol{\Sigma}_{X}\right)^{-1}+\boldsymbol{\Sigma}_{f}^{-1}\right)^{-1}, \\
& \boldsymbol{\mu}_{\mathbf{f} \mid r e s t}=\boldsymbol{\Sigma}_{\mathbf{f} \mid r e s t}\left(\left(\gamma_{R}^{-1} \boldsymbol{\Sigma}+\boldsymbol{\Sigma}_{X}\right)^{-1} \sum_{i=1}^{N} z_{1 i}\left(\mathbf{X}_{\mathbf{i}}\left(\mathbf{h}_{\mathbf{i}}\right)-z_{0 i} \mathbf{1}_{p}\right)\right) \\
& \sigma_{Y}^{2} \mid \text { rest } \sim \operatorname{IG}\left(a+N p / 2, b+1 / 2 \sum_{i=1}^{N}\left(\mathbf{Y}_{i}-\mathbf{X}_{i}\right)^{\prime}\left(\mathbf{Y}_{i}-\mathbf{X}_{i}\right)\right) \\
& \eta_{X} \mid \text { rest } \sim G\left(c+N, d+1 / 2 \sum_{i=1}^{N} \operatorname{tr}\left(\left(\mathbf{X}_{\mathbf{i}}\left(\mathbf{h}_{\mathbf{i}}\right)-\left(z_{0 i} \mathbf{1}_{p}+z_{1 i} \mathbf{f}\right)\right)\left(\mathbf{X}_{\mathbf{i}}\left(\mathbf{h}_{\mathbf{i}}\right)-\left(z_{0 i} \mathbf{1}_{p}+z_{1 i} \mathbf{f}\right)\right)^{\prime} \mathbf{P}_{1}^{-}\right),\right. \\
& \lambda_{X} \mid \text { rest } \sim G\left(c+N, d+1 / 2 \sum_{i=1}^{N} \operatorname{tr}\left(\left(\mathbf{X}_{\mathbf{i}}\left(\mathbf{h}_{\mathbf{i}}\right)-\left(z_{0 i} \mathbf{1}_{p}+z_{1 i} \mathbf{f}\right)\right)\left(\mathbf{X}_{\mathbf{i}}\left(\mathbf{h}_{\mathbf{i}}\right)-\left(z_{0 i} \mathbf{1}_{p}+z_{1 i} \mathbf{f}\right)\right)^{\prime} \mathbf{P}_{2}^{-}\right),\right. \\
& z_{0 i} \mid \text { rest } \sim N\left(\mu_{z_{0 i} \mid \text { rest }}, \sigma_{z_{0 i} \mid \text { rest }}^{2}\right) \text {, } \\
& \sigma_{z_{0 i} \mid \text { rest }}^{2}=\left(\sigma_{z_{0}}^{-2}+2 * \mathbf{1}_{p}^{\prime}\left(\lambda_{R}^{-1} \boldsymbol{\Sigma}+\boldsymbol{\Sigma}_{X}\right)^{-1} \mathbf{1}_{p}\right)^{-1}, \\
& \left.\mu_{z_{0 i} \mid \text { rest }}=\sigma_{z_{0 i} \mid \text { rest }}^{2}\left(\mathbf{X}_{\mathbf{i}}\left(\mathbf{h}_{\mathbf{i}}\right)-\mathbf{X}_{\mathbf{N}}\left(\mathbf{h}_{\mathbf{N}}\right)+\left(z_{1 N}-z_{1 i}\right) \mathbf{f}-\sum_{j=1}^{N-1} z_{0 j} \mathbf{1}\{j \neq i\} \mathbf{1}_{p}\right)^{\prime}\left(\gamma_{R}^{-1} \boldsymbol{\Sigma}+\boldsymbol{\Sigma}_{X}\right)^{-1} \mathbf{1}_{p}\right) \text {, } \\
& \sigma_{z_{0}}^{2} \mid \text { rest } \sim \operatorname{IG}\left(a+(N-1) / 2, b+1 / 2 \sum_{i=1}^{N-1} z_{0 i}^{2}\right), \\
& z_{1 i} \mid \text { rest } \sim N\left(\mu_{z_{1 i} \mid \text { rest }}, \sigma_{z_{1 i} \mid \text { rest }}^{2}\right) \text {, } \\
& \sigma_{z_{1 i} \mid r e s t}^{2}=\left(\sigma_{z_{1}}^{-2}+\mathbf{f}^{\prime}\left(\gamma_{R}^{-1} \boldsymbol{\Sigma}+\boldsymbol{\Sigma}_{X}\right)^{-1} \mathbf{f}\right)^{-1}, \\
& \mu_{z_{1 i} \mid \text { rest }}=\sigma_{z_{1 i} \mid \text { rest }}^{2}\left(\left(\mathbf{X}_{\mathbf{i}}\left(\mathbf{h}_{\mathbf{i}}\right)-z_{0 i} \mathbf{1}_{p}\right)^{\prime}\left(\lambda_{R}^{-1} \boldsymbol{\Sigma}+\boldsymbol{\Sigma}_{X}\right)^{-1} \mathbf{f}\right),
\end{aligned}
$$




$$
\begin{aligned}
\sigma_{z_{1}}^{2} \mid \text { rest } & \sim I G\left(a+N / 2, b+1 / 2 \sum_{i=1}^{N} z_{1 i}^{2}\right), \\
\eta_{f} \mid \text { rest } & \sim G\left(c+1, d+\left(\mathbf{f}^{\prime} \mathbf{P}_{1}^{-} \mathbf{f}\right) / 2\right), \text { and } \\
\lambda_{f} \mid \text { rest } & \sim G\left(c+(p-2) / 2, d+\left(\mathbf{f}^{\prime} \mathbf{P}_{2}^{-} \mathbf{f}\right) / 2\right) .
\end{aligned}
$$

Note, this list does not include a full conditional for the base functions or registered functions as their priors are not conjugate. Instead, the base and registered functions are sampled via a Metropolis step.

\section{A.3 Forcing Registered Time to Equal Standard Time}

1. If the functions are registered so that registered features occur at the average time that they appear in the unregistered sample, for all $t, t \in\left\{t_{1}, \ldots, t_{p}\right\}$, the average warping at that time point, $\overline{h .(t)}=\frac{1}{N} \sum_{i=1}^{N} h_{i}(t)$, is the identity. Over all observed time points this implies $\overline{\mathbf{h} .}=\left(\overline{h \cdot\left(t_{1}\right)}=t_{1}, \ldots, \overline{h \cdot\left(t_{p}\right)}=t_{p}\right)^{\prime}$.

2. Generally, after the registration process, this property will not hold, and instead $\overline{\mathbf{h} .}=\left(\overline{h .\left(t_{1}\right)}=\tilde{t}_{1}, \ldots, \overline{h .\left(t_{p}\right)}=\tilde{t}_{p}\right)^{\prime}$ where $t_{j} \neq \tilde{t}_{j}$ for at least one $j \in\{1, \ldots, p\}$.

3. The goal is to shift the functions so that these average warpings correspond to the correct registered times, i.e. $\tilde{\tilde{\mathbf{h}}} .=\left(\overline{h \cdot\left(\tilde{t}_{1}\right)}=\tilde{t}_{1}, \ldots, \overline{h \cdot\left(\tilde{t}_{p}\right)}=\tilde{t}_{p}\right)^{\prime}$.

4. (3) implies that after the initial registration, we have the correct registered function values over the new set of times, $\tilde{\mathbf{t}}=\left(\tilde{t}_{1}, \ldots, \tilde{t}_{p}\right)^{\prime}$, i.e. we have estimates of $\mathbf{X}_{i}\left(\tilde{\mathbf{h}}_{i}\right)=\left(X_{i}\left(h_{i}\left(\tilde{t}_{1}\right)\right), \ldots, X_{i}\left(h_{i}\left(\tilde{t}_{p}\right)\right)\right)^{\prime}$, for all $i=1, \ldots, N$.

5. If it is desired, the estimated values of the registered functions at the original time points, $\mathbf{t}$, can be obtained by interpolating values between the new set of time points, $\tilde{\mathbf{t}}$.

For example, after the initial registration process, suppose $\overline{h .(2)}=2.25$ and $\overline{h .(3)}=$ 3.1 , where 2 and 3 are in the set of original time points. We can alter registered time so that $\overline{h .(2.25)}=2.25$ and $\overline{h .(3.1)}=3.1$, as desired. Using the notation above, this implies $\{2.25,3.1\} \subset \tilde{\mathbf{t}}$ and for all $i, i=1 \ldots, N$, from the initial registration, we estimated the following values, $X_{i}\left(h_{i}(2.25)\right)$ and $X_{i}\left(h_{i}(3.1)\right)$. From these we can estimate $X_{i}\left(h_{i}(3)\right)$ by interpolating the values $X_{i}\left(h_{i}(2.25)\right)$ and $X_{i}\left(h_{i}(3.1)\right)$.

\section{Appendix B}

\section{B.1 Adapted Variational Bayes}

Below are the approximate posterior distributions for the adapted variational Bayes estimation procedure outlined in Section 3.1. 
Based on the full conditional distributions found in Appendix A.2, the following approximate posterior distributions are updated in each iteration for the registration model that accounts for noisy observations:

$$
\begin{aligned}
q\left(\mathbf{X}_{i}\right) & \sim N_{p}\left(\boldsymbol{\mu}_{q\left(\mathbf{X}_{i}\right)}, \boldsymbol{\Sigma}_{q\left(\mathbf{X}_{i}\right)}\right), \\
q(\mathbf{f}) & \sim N_{p}\left(\boldsymbol{\mu}_{q(\mathbf{f})}, \boldsymbol{\Sigma}_{q(\mathbf{f})}\right), \\
q\left(\sigma_{Y}^{2}\right) & \sim I G\left(a_{q\left(\sigma_{Y}^{2}\right)}, b_{q\left(\sigma_{Y}^{2}\right)}\right), \\
q\left(\eta_{X}\right) & \sim G\left(c_{q\left(\eta_{X}\right)}, d_{q\left(\eta_{X}\right)}\right), \\
q\left(\lambda_{X}\right) & \sim G\left(c_{q\left(\lambda_{X}\right)}, d_{q\left(\lambda_{X}\right)}\right), \\
q\left(z_{0 i}\right) & \sim N\left(\mu_{q\left(z_{0 i}\right)}, \sigma_{q\left(z_{0 i}\right)}^{2}\right) \\
q\left(\sigma_{z_{0}}^{2}\right) & \sim I G\left(a_{q\left(\sigma_{z_{0}}^{2}\right)}, b_{q\left(\sigma_{z_{0}}^{2}\right)}\right), \\
q\left(z_{1 i}\right) & \sim N\left(\mu_{q\left(z_{1 i}\right)}, \sigma_{q\left(z_{1 i}\right)}^{2}\right), \\
q\left(\sigma_{z_{1}}^{2}\right) & \sim I G\left(a_{q\left(\sigma_{z_{1}}^{2}\right)}, b_{q\left(\sigma_{z_{1}}^{2}\right)}\right), \\
q\left(\eta_{f}\right) & \sim G\left(c_{q\left(\eta_{f}\right)}, d_{q\left(\eta_{f}\right)}\right), \text { and } \\
q\left(\lambda_{f}\right) & \sim G\left(c_{q\left(\lambda_{f}\right)}, d_{q\left(\lambda_{f}\right)}\right) .
\end{aligned}
$$

If the observations are recorded without noise, i.e. we have observations $\mathbf{X}_{i}, i=$ $1 \ldots N$ as described in (5), the following $q$ distributions are omitted,

$$
q\left(\mathbf{X}_{i}\right) \text { for } i=1 \ldots N, q\left(\sigma_{y}^{2}\right), q\left(\eta_{X}\right) \text {, and } q\left(\lambda_{X}\right) .
$$

As the $q$ densities are all of known distributional forms, updating these densities is equivalent to updating their parameters. First, assuming the data are recorded without noise, for each iteration, the following parameters are updated for the $q$ densities listed above:

$$
\begin{aligned}
& \boldsymbol{\Sigma}_{q(\mathbf{f})}=\left[\sum_{i=1}^{N}\left(\sigma_{q\left(z_{1 i}\right)}^{2}+\mu_{q\left(z_{1 i}\right)}^{2}\right) \gamma_{R} \boldsymbol{\Sigma}^{-1}+\mu_{q\left(\eta_{\mathbf{f}}\right)} \mathbf{P}_{1}^{-}+\mu_{q\left(\lambda_{\mathbf{f}}\right)} \mathbf{P}_{2}^{-}\right]^{-1} \\
& \boldsymbol{\mu}_{q(\mathbf{f})}=\boldsymbol{\Sigma}_{q(\mathbf{f})} \gamma_{R} \boldsymbol{\Sigma}^{-1}\left[\sum_{i=1}^{N} \mu_{q\left(z_{1 i}\right)}\left(\mathbf{X}_{i}\left(\mathbf{h}_{i}\right)-\mu_{q\left(z_{0 i}\right)} \mathbf{1}_{p}\right)\right] \\
& \sigma_{q\left(z_{0 i}\right)}^{2}=\left(\mu_{q\left(\sigma_{z_{0}}^{-2}\right)}+2 \mathbf{1}_{p}^{\prime} \gamma_{R} \boldsymbol{\Sigma}^{-1} \mathbf{1}_{p}\right)^{-1} \\
& \mu_{q\left(z_{0 i}\right)}=\left[\sigma_{q\left(z_{0 i}\right)}^{2}\left(\mathbf{X}_{i}\left(\mathbf{h}_{i}\right)^{\prime}-\mathbf{X}_{N}\left(\mathbf{h}_{N}\right)^{\prime}+\left(\mu_{q\left(z_{1 N}\right)}-\mu_{q\left(z_{1 i}\right)}\right) \mu_{q(\mathbf{f})}^{\prime}-\sum_{j=1}^{N-1} \mu_{q\left(z_{0 j}\right)} \mathbf{1}\{i \neq j\} \mathbf{1}_{p}^{\prime}\right)\right] \gamma_{R} \boldsymbol{\Sigma}^{-1} \mathbf{1}_{p}, \\
& \sigma_{q\left(z_{1 i}\right)}^{2}=\left(\mu_{q\left(\sigma_{z_{1}}^{-2}\right)}+\operatorname{tr}\left(\left(\boldsymbol{\Sigma}_{q(\mathbf{f})}+\mu_{q(\mathbf{f})} \mu_{q(\mathbf{f})}^{\prime}\right) \gamma_{R} \boldsymbol{\Sigma}^{-1}\right)\right)^{-1} \\
& \mu_{q\left(z_{1 i}\right)}=\sigma_{q\left(z_{1 i}\right)}^{2}\left(\mu_{q\left(\sigma_{z_{1}}^{-2}\right)}+\mu_{q(\mathbf{f})}^{\prime} \gamma_{R} \boldsymbol{\Sigma}^{-1}\left(\mathbf{X}_{i}\left(\mathbf{h}_{i}\right)-\mu_{q\left(z_{0 i}\right)} \mathbf{1}_{p}\right)\right) \\
& d_{q\left(\eta_{\mathbf{f}}\right)}=d+1 / 2 * \operatorname{tr}\left(\mathbf{P}_{1}^{-}\left(\Sigma_{q(\mathbf{f})}+\mu_{q(\mathbf{f})} \mu_{q(\mathbf{f})}^{\prime}\right)\right)
\end{aligned}
$$


$d_{q\left(\lambda_{\mathbf{f}}\right)}=d+1 / 2 * \operatorname{tr}\left(\mathbf{P}_{2}^{-}\left(\Sigma_{q(\mathbf{f})}+\mu_{q(\mathbf{f})} \mu_{q(\mathbf{f})}^{\prime}\right)\right)$,

$b_{q\left(\sigma_{z_{0}}^{2}\right)}=b+1 / 2 \sum_{i=1}^{N-1}\left(\sigma_{q\left(z_{0 i}\right)}^{2}+\mu_{q\left(z_{0 i}\right)}^{2}\right)$, and

$b_{q\left(\sigma_{z_{1}}^{2}\right)}=b+1 / 2 \sum_{i=1}^{N}\left(\sigma_{q\left(z_{1 i}\right)}^{2}+\mu_{q\left(z_{1 i}\right)}^{2}\right)$.

Note, these updates are listed in an order that allows the convergence criterion to be calculated. Further details on the convergence criterion can be found in the next section.

For the model where observations are recorded with noise, in all of the updates above $\mathbf{X}_{i}\left(\mathbf{h}_{i}\right)$ and $\mathbf{X}_{N}\left(\mathbf{h}_{N}\right)$ are replaced by $\boldsymbol{\mu}_{q\left(\mathbf{X}_{i}\left(\mathbf{h}_{i}\right)\right)}$ and $\boldsymbol{\mu}_{q\left(\mathbf{X}_{N}\left(\mathbf{h}_{N}\right)\right)}$, respectively. Additionally, $\gamma_{R} \boldsymbol{\Sigma}^{-1}$ is replaced by $\left(\gamma_{R}^{-1} \boldsymbol{\Sigma}+\boldsymbol{\Sigma}_{X}\right)^{-1}$. For each $i, \boldsymbol{\mu}_{q\left(\mathbf{X}_{i}\left(\mathbf{h}_{i}\right)\right)}$ is determined by using the update for the mean of the $q$ distribution of the unregistered function, $\boldsymbol{\mu}_{q\left(\mathbf{X}_{i}\right)}$ below, and registering it using the current value of $\mathbf{h}_{i}$. In addition to these modified updates, the following additional updates necessary:

$$
\begin{aligned}
\boldsymbol{\Sigma}_{q\left(\mathbf{X}_{i}\right)} & =\left(\mu_{q\left(\frac{1}{\sigma_{Y}^{2}}\right)} \mathbf{I}_{p}+\mu_{q\left(\eta_{X}\right)} \mathbf{P}_{1}^{-}+\mu_{q\left(\lambda_{X}\right)} \mathbf{P}_{2}^{-}\right)^{-1} \\
\boldsymbol{\mu}_{q\left(\mathbf{X}_{i}\right)}= & \boldsymbol{\Sigma}_{q\left(\mathbf{X}_{i}\right)}\left[\mu_{q\left(\frac{1}{\sigma_{Y}^{2}}\right)} \mathbf{Y}_{i}+\left(\mu_{q\left(\eta_{X}\right)} \mathbf{P}_{1}^{-}+\mu_{q\left(\lambda_{X}\right)} \mathbf{P}_{2}^{-}\right)\left(\mu_{q\left(z_{0 i}\right)} \mathbf{1}_{p}+\mu_{q\left(z_{1 i}\right)} E_{\left(\theta_{\left.-\mathbf{x}_{i}\right)}\right)}\left[\mathbf{f}\left(\mathbf{h}_{i}^{-1}\right)\right]\right)\right] \\
b_{q\left(\sigma_{Y}^{2}\right)}= & b+\frac{1}{2} \sum_{i=1}^{N}\left(\mathbf{Y}_{i}^{\prime} \mathbf{Y}_{i}-2 \boldsymbol{\mu}_{q\left(\mathbf{X}_{i}\right)}^{\prime} \mathbf{Y}_{i}+\sum_{j=1}^{p} \boldsymbol{\Sigma}_{q\left(\mathbf{X}_{i}\right)}[j, j]+\boldsymbol{\mu}_{q\left(\mathbf{X}_{i}\right)}[j]^{2}\right) \\
d_{q\left(\eta_{\mathbf{X}}\right)}= & d+\frac{1}{2} \operatorname{tr}\left[\sum _ { i = 1 } ^ { N } \left(\boldsymbol{\Sigma}_{q\left(\mathbf{X}_{i}\right)}+\boldsymbol{\mu}_{q\left(\mathbf{X}_{i}\right)} \boldsymbol{\mu}_{q\left(\mathbf{X}_{i}\right)}^{\prime}-2 \boldsymbol{\mu}_{q\left(\mathbf{X}_{i}\right)}\left(\mu_{q\left(z_{0 i}\right)} \mathbf{1}_{p}+\mu_{q\left(z_{1 i}\right)} E_{\left(\theta_{-\eta_{X}}\right)}\left[\mathbf{f}\left(\mathbf{h}_{i}^{-1}\right)\right]\right)^{\prime}\right.\right. \\
& +\left(\sigma_{q\left(z_{0 i}\right)}^{2}+\mu_{q\left(z_{0 i}\right)}^{2}\right) \mathbf{1}_{p} \mathbf{1}_{p}^{\prime}+2 \mu_{q\left(z_{0 i}\right)} \mu_{q\left(z_{1 i}\right)} \mathbf{1}_{p} E_{\left(\theta_{\left.-\eta_{X}\right)}\right)}\left[\mathbf{f}\left(\mathbf{h}_{i}^{-1}\right)\right]^{\prime} \\
& \left.\left.+\left(\sigma_{q\left(z_{1 i}\right)}^{2}+\mu_{q\left(z_{1 i}\right)}^{2}\right) E_{\left(\theta_{-\eta_{X}}\right)}\left[\mathbf{f}\left(\mathbf{h}_{i}^{-1}\right) \mathbf{f}\left(\mathbf{h}_{i}^{-1}\right)^{\prime}\right]\right) \mathbf{P}_{1}^{-}\right], \text {and } \\
d_{q\left(\lambda_{\mathbf{X}}\right)}= & d+\frac{1}{2} \operatorname{tr}\left[\sum _ { i = 1 } ^ { N } \left(\boldsymbol{\Sigma}_{q\left(\mathbf{X}_{i}\right)}+\boldsymbol{\mu}_{q\left(\mathbf{X}_{i}\right)} \boldsymbol{\mu}_{q\left(\mathbf{X}_{i}\right)}^{\prime}-2 \boldsymbol{\mu}_{q\left(\mathbf{X}_{i}\right)}\left(\mu_{q\left(z_{0 i}\right)} \mathbf{1}_{p}+\mu_{q\left(z_{1 i}\right)} E_{\left(\theta_{\left.-\lambda_{X}\right)}\right)}\left[\mathbf{f}\left(\mathbf{h}_{i}^{-1}\right)\right]\right)^{\prime}\right.\right. \\
& +\left(\sigma_{q\left(z_{0 i}\right)}^{2}+\mu_{q\left(z_{0 i}\right)}^{2}\right) \mathbf{1}_{p} \mathbf{1}_{p}^{\prime}+2 \mu_{q\left(z_{0 i}\right)} \mu_{q\left(z_{1 i}\right)} \mathbf{1}_{p} E_{\left(\theta_{-\lambda_{X}}\right)}\left[\mathbf{f}\left(\mathbf{h}_{i}^{-1}\right)\right]^{\prime} \\
& \left.\left.+\left(\sigma_{q\left(z_{1 i}\right)}^{2}+\mu_{q\left(z_{1 i}\right)}^{2}\right) E_{\left(\theta_{\left.-\lambda_{X}\right)}\right)}\left[\mathbf{f}\left(\mathbf{h}_{i}^{-1}\right) \mathbf{f}\left(\mathbf{h}_{i}^{-1}\right)^{\prime}\right]\right) \mathbf{P}_{2}^{-}\right] .
\end{aligned}
$$

Note, these updates contain terms that cannot be evaluated. For instance, $E_{\left(\theta_{-\eta_{X}}\right)}\left[\mathbf{f}\left(\mathbf{h}_{i}^{-1}\right) \mathbf{f}\left(\mathbf{h}_{i}^{-1}\right)^{\prime}\right]$ cannot be determined because the approximate distribution of $\mathbf{f}\left(\mathbf{h}_{i}^{-1}\right)$ is unknown. These terms can however be approximated. Appendix C.2 provides details of the approximated values used for this analysis. 


\section{B.2 Convergence Criterion}

When the functional observations, $\mathbf{X}=\mathbf{X}_{i}, i \ldots N$, are recorded without noise, $E_{q\left(\boldsymbol{\theta}_{-\mathbf{w}}\right)}\left[\log f\left(\mathbf{X}, \mathbf{w}, \boldsymbol{\theta}_{-\mathbf{w}}\right)-\log q\left(\boldsymbol{\theta}_{-\mathbf{w}}\right)\right]$ is monitored until the desired threshhold is met, where

$$
\begin{aligned}
E_{q\left(\boldsymbol{\theta}_{-\mathbf{w}}\right)}\left[\log f\left(\mathbf{X}, \mathbf{w}, \boldsymbol{\theta}_{-\mathbf{w}}\right)-\log q\left(\boldsymbol{\theta}_{-\mathbf{w}}\right)\right]= & E_{q\left(\boldsymbol{\theta}_{-\mathbf{w}}\right)}\left[\log \left(f\left(\mathbf{X}, \mathbf{w} \mid \boldsymbol{\theta}_{-\mathbf{w}}\right) f\left(\boldsymbol{\theta}_{-\mathbf{w}}\right)\right)-\log q\left(\boldsymbol{\theta}_{-\mathbf{w}}\right)\right] \\
= & E_{q\left(\boldsymbol{\theta}_{-\mathbf{w}}\right)}\left[\log f\left(\mathbf{X}, \mathbf{w} \mid \boldsymbol{\theta}_{-\mathbf{w}}\right)+\log f\left(\boldsymbol{\theta}_{-\mathbf{w}}\right)-\log q\left(\boldsymbol{\theta}_{-\mathbf{w}}\right)\right] \\
= & E_{q\left(\boldsymbol{\theta}_{-\mathbf{w}}\right)}\left[\log f\left(\mathbf{X}, \mathbf{w} \mid \boldsymbol{\theta}_{-\mathbf{w}}\right)\right] \\
& +E_{q\left(\boldsymbol{\theta}_{-\mathbf{w}}\right)}[\log f(\mathbf{f})-\log q(\mathbf{f})] \\
& +\sum_{i=1}^{(N-1)} E_{q\left(\boldsymbol{\theta}_{-\mathbf{w}}\right)}\left[\log f\left(z_{0 i}\right)-\log q\left(z_{0 i}\right)\right] \\
& +\sum_{i=1}^{(N)} E_{q\left(\boldsymbol{\theta}_{-\mathbf{w}}\right)}\left[\log f\left(z_{1 i}\right)-\log q\left(z_{1 i}\right)\right] \\
& +E_{q\left(\boldsymbol{\theta}_{-\mathbf{w}}\right)}\left[\log f\left(\sigma_{z_{0}}^{2}\right)-\log q\left(\sigma_{z_{0}}^{2}\right)\right] \\
& +E_{q\left(\boldsymbol{\theta}_{-\mathbf{w}}\right)}\left[\log f\left(\sigma_{z_{1}}^{2}\right)-\log q\left(\sigma_{z_{1}}^{2}\right)\right] \\
& +E_{q\left(\boldsymbol{\theta}_{-\mathbf{w}}\right)}\left[\log f\left(\eta_{f}\right)-\log q\left(\eta_{f}\right)\right] \\
& +E_{q\left(\boldsymbol{\theta}_{-\mathbf{w}}\right)}\left[\log f\left(\lambda_{f}\right)-\log q\left(\lambda_{f}\right)\right]
\end{aligned}
$$

Now looking at each piece individually,

$$
\begin{aligned}
E_{q\left(\boldsymbol{\theta}_{-\mathbf{w}}\right)}\left[\log f\left(\mathbf{X}, \mathbf{w} \mid \boldsymbol{\theta}_{-\mathbf{w}}\right)\right] & E_{q\left(\boldsymbol{\theta}_{-\mathbf{w}}\right)}\left[\sum_{i=1}^{N}\left(\log \left[(2 \pi)^{-p / 2}\left|\gamma_{R}^{-1} \boldsymbol{\Sigma}\right|^{-1 / 2}\right]\right)\right] \\
& +E_{q\left(\boldsymbol{\theta}_{-\mathbf{w}}\right)}\left[\sum_{i=1}^{N}-\frac{1}{2}\left[\left(\mathbf{X}_{i}\left(\mathbf{h}_{i}\right)-\left(z_{0 i} \mathbf{1}_{p}+z_{1 i} \mathbf{f}\right)\right)^{\prime} \gamma_{R} \boldsymbol{\Sigma}^{-1}\left(\mathbf{X}_{i}\left(\mathbf{h}_{i}\right)-\left(z_{0 i} \mathbf{1}_{p}+z_{1 i} \mathbf{f}\right)\right)\right]\right] \\
= & \sum_{i=1}^{N}\left(\log \left[(2 \pi)^{-p / 2}\left|\gamma_{R}^{-1} \boldsymbol{\Sigma}\right|^{-1 / 2}\right]\right) \\
& +\sum_{i=1}^{N}-\frac{1}{2}\left[\left(\mathbf{X}_{i}\left(\mathbf{h}_{i}\right)^{\prime} \gamma_{R} \boldsymbol{\Sigma}^{-1} \mathbf{X}_{i}\left(\mathbf{h}_{i}\right)\right)-\right. \\
& 2 \mathbf{X}_{i}\left(\mathbf{h}_{i}\right)^{\prime} \gamma_{R} \boldsymbol{\Sigma}^{-1} \mu_{q\left(z_{0 i}\right)} \mathbf{1}_{p}-2 \mathbf{X}_{i}\left(\mathbf{h}_{i}\right)^{\prime} \gamma_{R} \boldsymbol{\Sigma}^{-1} \mu_{q\left(z_{1 i}\right)} \boldsymbol{\mu}_{q(\mathbf{f})}+ \\
& \left(\sigma_{q\left(z_{1 i}\right)}^{2}+\mu_{q\left(z_{1 i}\right)}^{2}\right) \operatorname{tr}\left(\left(\boldsymbol{\Sigma}_{q(\mathbf{f})}+\boldsymbol{\mu}_{q(\mathbf{f})} \boldsymbol{\mu}_{q(\mathbf{f})}^{\prime}\right) \gamma_{R} \boldsymbol{\Sigma}^{-1}\right)+ \\
& \left.2 \mu_{q\left(z_{0 i}\right)} \mu_{q\left(z_{1 i}\right)} \mathbf{1}_{p}^{\prime} \gamma_{R} \boldsymbol{\Sigma}^{-1} \boldsymbol{\mu}_{q(\mathbf{f})}\right]- \\
& {\left[\sum_{i=1}^{N-1}\left(\sigma_{q\left(z_{0 i}\right)}^{2}+\mu_{q\left(z_{0 i}\right)}^{2}\right)+\frac{1}{2} \sum_{i=1}^{N-1} \sum_{j=1}^{N-1} \mu_{q\left(z_{0 i}\right)} \mu_{q\left(z_{0 j}\right)} \mathbf{1}\{j \neq i\}\right] \mathbf{1}_{p}^{\prime} \gamma_{R} \boldsymbol{\Sigma}^{-1} \mathbf{1}_{p}, }
\end{aligned}
$$




$$
\begin{aligned}
E_{q\left(\boldsymbol{\theta}_{-\mathbf{w}}\right)}[\log f(\mathbf{f})-\log q(\mathbf{f})]= & E_{q\left(\boldsymbol{\theta}_{-\mathbf{w}}\right)}\left[-\frac{p}{2} \log 2 \pi+\frac{1}{2} \log \left|\eta_{f} \mathbf{P}_{1}^{-}+\lambda_{f} \mathbf{P}_{2}^{-}\right|\right]- \\
& E_{q\left(\boldsymbol{\theta}_{-\mathbf{w}}\right)}\left[\frac{1}{2}\left(\operatorname{tr}\left[\mathbf{f f}^{\prime}\left(\eta_{f} \mathbf{P}_{1}^{-}+\lambda_{f} \mathbf{P}_{2}^{-}\right)\right]\right]+\right. \\
& E_{q\left(\boldsymbol{\theta}_{-\mathbf{w}}\right)}\left[\frac{p}{2} \log 2 \pi+\frac{1}{2} \log \left|\boldsymbol{\Sigma}_{q(\mathbf{f})}\right|\right]+ \\
& E_{q\left(\boldsymbol{\theta}_{-\mathbf{w}}\right)}\left[\frac{1}{2} \operatorname{tr}\left(\mathbf{f f}^{\prime} \boldsymbol{\Sigma}_{q(\mathbf{f})}^{-1}\right)-\mathbf{f}^{\prime} \boldsymbol{\Sigma}_{q(\mathbf{f})}^{-1} \boldsymbol{\mu}_{q(\mathbf{f})}\right]+ \\
& E_{q\left(\boldsymbol{\theta}_{-\mathbf{w}}\right)}\left[\frac{1}{2} \boldsymbol{\mu}_{q(\mathbf{f})}^{\prime} \boldsymbol{\Sigma}_{q(\mathbf{f})}^{-1} \boldsymbol{\mu}_{q(\mathbf{f})}\right] \\
= & C+\frac{1}{2} E_{q\left(\boldsymbol{\theta}_{-\mathbf{w}}\right)}\left[2 \log \eta_{f}\right]+\frac{1}{2} E_{q\left(\boldsymbol{\theta}_{-\mathbf{w}}\right)}\left[(p-2) \log \lambda_{f}\right]- \\
& \frac{1}{2} \operatorname{tr}\left(\left(\boldsymbol{\Sigma}_{q(\mathbf{f})}+\boldsymbol{\mu}_{q(\mathbf{f})} \boldsymbol{\mu}_{q(\mathbf{f})}^{\prime}\right)\left(\mu_{q\left(\eta_{f}\right)} \mathbf{P}_{1}^{-}+\mu_{q\left(\lambda_{f}\right)} \mathbf{P}_{2}^{-}\right)\right)- \\
& \frac{1}{2} \log \left|\boldsymbol{\Sigma}_{q(\mathbf{f})}^{-1}\right|+\frac{p}{2},
\end{aligned}
$$

where $C$ is a constant that does not change from one iteration to the next. For $\mathbf{z}_{0}=$ $\left(z_{01}, \ldots, z_{0(N-1)}\right)^{\prime}$,

$$
\begin{aligned}
E_{q\left(\boldsymbol{\theta}_{-\mathbf{w}}\right)}\left[\log f\left(\mathbf{z}_{0}\right)-\log q\left(\mathbf{z}_{0}\right)\right]= & E_{q\left(\boldsymbol{\theta}_{-\mathbf{w}}\right)}\left[-\frac{N-1}{2} \log 2 \pi-\frac{N-1}{2} \log \sigma_{z_{0}}^{2}-\sum_{i=1}^{N-1}-\frac{1}{2 \sigma_{z_{0}}^{2}} z_{0 i}^{2}+\right. \\
& \frac{N-1}{2} \log 2 \pi+\frac{N-1}{2} \log \sigma_{q\left(z_{0 i}\right)}^{2}+ \\
& \left.\sum_{i=1}^{N-1} \frac{1}{2 \sigma_{q\left(z_{0 i}\right)}^{2}}\left(z_{0 i}-\mu_{q\left(z_{0 i}\right)}\right)^{2}\right] \\
= & \frac{N-1}{2} \log \sigma_{q\left(z_{0 i}\right)}^{2}-E_{q\left(\boldsymbol{\theta}_{-\mathbf{w}}\right)}\left[\frac{N-1}{2} \log \sigma_{z_{0}}^{2}\right]- \\
& \frac{1}{2} \mu_{q\left(\frac{1}{\sigma_{z_{0}}^{2}}\right)}\left(\sum_{i=1}^{N-1}\left(\sigma_{q\left(z_{0 i}\right)}^{2}+\mu_{q\left(z_{0 i}\right)}^{2}\right)\right)+\frac{N-1}{2} .
\end{aligned}
$$

For $\mathbf{z}_{1}=\left(z_{11}, \ldots, z_{1 N}\right)^{\prime}$

$$
\begin{aligned}
E_{q\left(\boldsymbol{\theta}_{-\mathbf{w}}\right)}\left[\log f\left(\mathbf{z}_{1}\right)-\log q\left(\mathbf{z}_{1}\right)\right]= & E_{q\left(\boldsymbol{\theta}_{-\mathbf{w}}\right)}\left[-\frac{N}{2} \log 2 \pi-\frac{N}{2} \log \sigma_{z_{1}}^{2}-\sum_{i=1}^{N}-\frac{1}{2 \sigma_{z_{1}}^{2}} z_{1 i}^{2}+\right. \\
& \left.\frac{N}{2} \log 2 \pi+\frac{N}{2} \log \sigma_{q\left(z_{1}\right)}^{2}+\sum_{i=1}^{N} \frac{1}{2 \sigma_{q\left(z_{1 i}\right)}^{2}}\left(z_{1 i}-\mu_{q\left(z_{1 i}\right)}\right)^{2}\right] \\
= & \frac{N}{2} \log \sigma_{q\left(z_{1 i}\right)}^{2}-E_{q\left(\boldsymbol{\theta}_{-\mathbf{w}}\right)}\left[\frac{N}{2} \log \sigma_{z_{1}}^{2}\right]-
\end{aligned}
$$




$$
\frac{1}{2} \mu_{q\left(\frac{1}{\sigma_{z_{1}}^{2}}\right)}\left(\sum_{i=1}^{N}\left(\sigma_{q\left(z_{1 i}\right)}^{2}+\mu_{q\left(z_{1 i}\right)}^{2}\right)\right)+\frac{N}{2}
$$

For $\sigma_{z_{0}}^{2}$,

$$
\begin{aligned}
E_{q\left(\boldsymbol{\theta}_{-\mathbf{w}}\right)}\left[\log f\left(\sigma_{z_{0}}^{2}\right)-\log q\left(\sigma_{z_{0}}^{2}\right)\right]= & E_{q\left(\boldsymbol{\theta}_{-\mathbf{w}}\right)}\left[\log \frac{b^{a}}{\Gamma(a)}-(a+1) \log \sigma_{z_{0}}^{2}-b \frac{1}{\sigma_{z_{0}}^{2}}-\right. \\
& \log \frac{b_{q\left(\sigma_{z_{0}}^{2}\right)}^{a_{q\left(z_{0}^{2}\right)}}}{\Gamma\left(a_{q\left(\sigma_{z_{0}}^{2}\right)}\right)}+\left(a_{q\left(\sigma_{z_{0}}^{2}\right)}+1\right) \log \sigma_{z_{0}}^{2}+ \\
& \left.b_{q\left(\sigma_{z_{0}}^{2}\right)} \frac{1}{\sigma_{z_{0}}^{2}}\right] \\
= & E_{q\left(\boldsymbol{\theta}_{-\mathbf{w}}\right)}\left[-(a+1) \log \sigma_{z_{0}}^{2}\right]-b \mu_{q\left(\frac{1}{\sigma_{z_{0}}^{2}}\right)}-\log \frac{\left.b_{q\left(\sigma_{z_{0}}^{2}\right)}^{a_{q\left(\sigma_{0}^{2}\right)}}+a_{q\left(\sigma_{z_{0}}^{2}\right)}\right)}{\Gamma} \\
& \log \frac{b^{a}}{\Gamma(a)}+E_{q\left(\boldsymbol{\theta}_{-\mathbf{w}}\right)}\left[\left(a_{q\left(\sigma_{z_{0}}^{2}\right)}+1\right) \log \sigma_{z_{0}}^{2}\right]+b_{q\left(\sigma_{z_{0}}^{2}\right)} \mu_{q\left(\frac{1}{\sigma_{z_{0}}^{2}}\right)} .
\end{aligned}
$$

For $\sigma_{z_{1}}^{2}$,

$$
\begin{aligned}
E_{q\left(\boldsymbol{\theta}_{-\mathbf{w}}\right)}\left[\log f\left(\sigma_{z_{1}}^{2}\right)-\log q\left(\sigma_{z_{1}}^{2}\right)\right]= & E_{q\left(\boldsymbol{\theta}_{-\mathbf{w}}\right)}\left[\log \frac{b^{a}}{\Gamma(a)}-(a+1) \log \sigma_{z_{1}}^{2}-b \frac{1}{\sigma_{z_{1}}^{2}}-\right. \\
& \log \frac{b_{q\left(\sigma_{z_{1}}^{2}\right)}^{\left.a_{\left(\sigma_{1}\right.}^{2}\right)}}{\Gamma\left(a_{q\left(\sigma_{z_{1}}^{2}\right)}\right)}+\left(a_{q\left(\sigma_{z_{1}}^{2}\right)}+1\right) \log \sigma_{z_{1}}^{2}+ \\
& \left.b_{q\left(\sigma_{z_{1}}^{2}\right)} \frac{1}{\sigma_{z_{1}}^{2}}\right] \\
= & E_{q\left(\boldsymbol{\theta}_{-\mathbf{w}}\right)}\left[-(a+1) \log \sigma_{z_{1}}^{2}\right]-b \mu_{q\left(\frac{1}{\sigma_{z_{1}}^{2}}\right)}-\log \frac{b_{q\left(\sigma_{z_{1}}^{2}\right)}^{\left.a_{q\left(\sigma_{1}\right.}^{2}\right)}}{\Gamma\left(a_{q\left(\sigma_{z_{1}}^{2}\right)}\right)}+ \\
& \log \frac{b^{a}}{\Gamma(a)}+E_{q\left(\boldsymbol{\theta}_{-\mathbf{w}}\right)}\left[\left(a_{q\left(\sigma_{z_{1}}^{2}\right)}+1\right) \log \sigma_{z_{1}}^{2}\right]+b_{q\left(\sigma_{z_{1}}^{2}\right)} \mu_{q\left(\frac{1}{\sigma_{z_{1}}^{2}}\right)} .
\end{aligned}
$$

For $\eta_{f}$,

$$
\begin{aligned}
E_{q\left(\boldsymbol{\theta}_{-\mathbf{w}}\right)}\left[\log f\left(\eta_{f}\right)-\log q\left(\eta_{f}\right)\right]= & E_{q\left(\boldsymbol{\theta}_{-\mathbf{w}}\right)}\left[\log \frac{d^{c}}{\Gamma(c)}+(c-1) \log \eta_{f}-d \eta_{f}-\right. \\
& \left.\log \frac{d_{q\left(\eta_{f}\right)}^{c_{q\left(\eta_{f}\right)}}}{\Gamma\left(c_{q\left(\eta_{f}\right)}\right)}-c \log \eta_{f}+d_{q\left(\eta_{f}\right)} \eta_{f}\right]
\end{aligned}
$$




$$
\begin{aligned}
= & \log \frac{d^{c}}{\Gamma(c)}-\log \frac{d_{q\left(\eta_{f}\right)}^{c_{q\left(\eta_{X}\right)}}}{\Gamma\left(c_{q\left(\eta_{f}\right)}\right)}-E_{q\left(\boldsymbol{\theta}_{-\mathbf{w}}\right)}\left[\log \eta_{f}\right]-d \mu_{q\left(\eta_{f}\right)}+ \\
& d_{q\left(\eta_{f}\right)} \mu_{q\left(\eta_{f}\right)} .
\end{aligned}
$$

For $\lambda_{f}$,

$$
\begin{aligned}
E_{q\left(\boldsymbol{\theta}_{-\mathbf{w}}\right)}\left[\log f\left(\lambda_{f}\right)-\log q\left(\lambda_{f}\right)\right]= & E_{q\left(\boldsymbol{\theta}_{-\mathbf{w}}\right)}\left[\log \frac{d^{c}}{\Gamma(c)}+(c-1) \log \lambda_{f}-d \lambda_{f}-\right. \\
& \left.\log \frac{d_{q\left(\lambda_{f}\right)}^{c_{q\left(\lambda_{f}\right)}}}{\Gamma\left(c_{q\left(\lambda_{f}\right)}\right)}-\left(\frac{p-2}{2}+c-1\right) \log \lambda_{f}+d_{q\left(\lambda_{f}\right)} \lambda_{f}\right] \\
= & \log \frac{d^{c}}{\Gamma(c)}-\log \frac{d_{q\left(\lambda_{f}\right)}^{c_{q\left(\lambda_{f}\right)}}}{\Gamma\left(c_{q\left(\lambda_{f}\right)}\right)}-\frac{p-2}{2} E_{q\left(\boldsymbol{\theta}_{-\mathbf{w}}\right)}\left[\log \lambda_{f}\right]-d \mu_{q\left(\lambda_{f}\right)}+ \\
& d_{q\left(\lambda_{f}\right)} \mu_{q\left(\lambda_{f}\right)} .
\end{aligned}
$$

The expression for $E_{q\left(\boldsymbol{\theta}_{-\mathbf{w}}\right)}\left[\log f\left(\mathbf{X}, \mathbf{w}, \boldsymbol{\theta}_{-\mathbf{w}}\right)-\log q\left(\boldsymbol{\theta}_{-\mathbf{w}}\right)\right]$ can be simplified much further by combining terms that cancel out. However, in some cases the ability to cancel terms depends on the order of the updates. For instance, in the expression, $E_{q\left(\boldsymbol{\theta}_{-\mathbf{w}}\right)}\left[\log f\left(\sigma_{z_{0}}^{2}\right)-\log q\left(\sigma_{z_{0}}^{2}\right)\right]$, the terms $-b \mu_{q\left(\frac{1}{\sigma_{z_{0}}^{2}}\right)}$ and $b_{q\left(\sigma_{z_{0}}^{2}\right)} \mu_{q\left(\frac{1}{\sigma_{z_{0}}^{2}}\right)}$ cancel with $-\frac{1}{2} \mu_{q\left(\frac{1}{\sigma_{z_{0}}^{2}}\right)}\left(\sum_{i=1}^{N-1}\left(\sigma_{q\left(z_{0 i}\right)}^{2}+\mu_{q\left(z_{0 i}\right)}^{2}\right)\right)$ from $E_{q\left(\boldsymbol{\theta}_{-\mathbf{w}}\right)}\left[\log f\left(\mathbf{z}_{0}\right)-\log q\left(\mathbf{z}_{0}\right)\right]$ as long as the parameters of $q\left(\mathbf{z}_{0}\right)$ are updated before $b_{q\left(\sigma_{z_{0}}^{2}\right)}$. For convenience, we have taken account the ordering necessary to compute the convergence criterion in the updates given above. Additionally, note all components in this expression that do not change from one iteration to the next can be ignored.

\section{Appendix C}

\section{C.1 Functional Data Regularization and Registration}

If instead of the function itself, noisy observations of each unregistered function, $X_{i}(t)$ are observed over a finite number of time points, $\mathbf{t}=\left(t_{1}, \ldots, t_{p}\right)^{\prime}$, we will additionally assume that the observations, $Y_{i}\left(t_{j}\right), j=1, \ldots, p$ are iid, $N\left(X_{i}\left(t_{j}\right), \sigma_{Y}^{2}\right)$. Incorporating registration and smoothing into a single model has also been considered recently by Raket et al. (2014). In their paper, each registered noisy function, $Y_{i}\left(h_{i}(t)\right)$ at time point $t_{j}, j=1, \ldots, p$ is composed as follows:

$$
Y_{i}\left(h_{i}\left(t_{j}\right)\right)=f\left(t_{j}\right)+r_{i}\left(t_{j}\right)+\epsilon_{i}\left(t_{j}\right),
$$

where $f(t)$ is similar to our target function, $r_{i}(t)$ is a function-specific random effect that accounts for variation in individual noiseless functions beyond the target function, and $\epsilon_{i}\left(t_{j}\right)$ are iid Gaussian noise. 
The advantage of our model is that incorporating individual random effects is unnecessary. Noting that the observations are noisy, not the registered functions; smoothing in our model is applied to the observations, not to the functions after registration. Under these conditions, variability in the estimated unregistered, smoothed functions, $X_{i}(t)$, can be looked at separately from variability in the estimated registered functions, $X_{i}\left(h_{i}(t)\right)$. Appendix C.3 provides an example of how treating smoothing as a pre-processing step underestimates variability in the posterior distributions of the registered functions.

In the presence of noisy observations, the following distributions are either altered or added to the registration model presented in Section 2,

$$
\begin{aligned}
Y_{i}\left(t_{j}\right) \mid X_{i}\left(t_{j}\right) & \sim N\left(X_{i}\left(t_{j}\right), \sigma_{Y}^{2}\right), \quad i=1 \ldots N, \quad j=1, \ldots, p \\
\mathbf{X}_{i}\left(\mathbf{h}_{i}\right) \mid z_{0 i}, z_{1 i}, \mathbf{f}, \eta_{X}, \lambda_{X} & \sim N_{p}\left(z_{0 i} \mathbf{1}+z_{1 i} \mathbf{f}, \gamma_{R}^{-1} \boldsymbol{\Sigma}+\boldsymbol{\Sigma}_{X}\right), \quad i=1, \ldots, N, \\
\boldsymbol{\Sigma}_{X} & =\eta_{X}^{-1} \mathbf{P}_{1}+\lambda_{X}^{-1} \mathbf{P}_{2}, \\
\eta_{X} & \sim G(c, d), \\
\lambda_{X} & \sim G(c, d), \text { and } \\
\sigma_{Y}^{2} & \sim I G(a, b) .
\end{aligned}
$$

The most significant change to the model is that we now include a smoothing penalty in the covariance specification for the registered functions. Here specifying $\mathbf{P}_{2}$ (a roughness penalty - see Section 2 for more details) in the prior distribution for the registered functions establishes regularization in these functions. The associated smoothing parameter is $\lambda_{X}$. As mentioned previously, $\mathbf{P}_{1}$ is required to define $\boldsymbol{\Sigma}_{X}$ as a proper covariance matrix. More details on these matrices can be found in Appendix A. As can be seen above, $\eta_{X}$ and $\lambda_{X}$ are considered as additional unknown parameters in this hierarchical model.

In the prior specifications of this model, equation (6) incorporates penalties for both smoothing and registration within the prior for the registered functions. The full conditional distribution for each approximated registered function, $\mathbf{X}_{i}\left(\mathbf{h}_{i}\right)$, when data are noisily observed is the joint full-conditional of the unregistered function and the warping function,

$$
\begin{aligned}
f\left(\mathbf{X}_{i}\left(\mathbf{h}_{i}\right) \mid \text { rest }\right) & =f\left(\mathbf{w}_{i}, \mathbf{X}_{i} \mid \text { rest }\right) \\
& =f\left(\mathbf{w}_{i} \mid \mathbf{X}_{i}, \text { rest }\right) f\left(\mathbf{X}_{i} \mid \text { rest }\right)
\end{aligned}
$$

Instead of drawing from this joint full-conditional directly, we will proceed by first drawing from $f\left(\mathbf{X}_{i} \mid\right.$ rest $)$ and then given $\mathbf{X}_{i}$, draw from $f\left(\mathbf{w}_{i} \mid \mathbf{X}_{i}\right.$, rest $)$.

These full conditional distributions are determined in the standard way recognizing that the prior distribution for a registered function can be factored into two components. One component penalizes lack of registration given the approximated unregistered function, $\mathbf{X}_{i}$; the other component penalizes roughness in the registered function 
which implicitly penalizes roughness in the unregistered function. The roughness penalty is independent of the warping function and therefore also of $\mathbf{w}_{i}$. Specifically, the prior distribution (6) for each $\mathbf{X}_{i}\left(\mathbf{h}_{i}\right), i=1, \ldots, N$, is such that

$$
\begin{aligned}
f\left(\mathbf{X}_{i}\left(\mathbf{h}_{i}\right) \mid \mathbf{X}_{i}, \mathbf{w}_{i}, z_{0 i}, z_{1 i}, \mathbf{f}, \eta_{X}, \lambda_{X}\right) \propto \\
\exp \left[-\frac{1}{2}\left(\left(\mathbf{X}_{i}\left(\mathbf{h}_{i}\right)-\left(z_{0 i} \mathbf{1}+z_{1 i} \mathbf{f}\right)\right)^{\prime} \gamma_{R} \boldsymbol{\Sigma}^{-1}\left(\mathbf{X}_{i}\left(\mathbf{h}_{i}\right)-\left(z_{0 i} \mathbf{1}+z_{1 i} \mathbf{f}\right)\right)\right)\right] * \\
\exp \left[-\frac{1}{2}\left(\left(\mathbf{X}_{i}\left(\mathbf{h}_{i}\right)-\left(z_{0 i} \mathbf{1}+z_{1 i} \mathbf{f}\right)\right)^{\prime} \boldsymbol{\Sigma}_{X}^{-1}\left(\mathbf{X}_{i}\left(\mathbf{h}_{i}\right)-\left(z_{0 i} \mathbf{1}+z_{1 i} \mathbf{f}\right)\right)\right)\right]
\end{aligned}
$$

Accordingly, the components of the joint distribution of the data and all unknown parameters that are dependent on $\mathbf{w}_{i}$ are (7), and the prior on the approximated base functions,

$$
\begin{aligned}
\mathbf{w}_{i} \propto \quad & N_{p-1}\left(\mathbf{0}, \gamma_{w}^{-1} \mathbf{\Sigma}+\lambda_{w}^{-1} \mathbf{P}_{w}\right) \mathbf{1}\left\{t_{1}+\sum_{k=2}^{p}\left(t_{k}-t_{k-1}\right) \exp \left(w_{i}\left(t_{k-1}\right)\right)=t_{p}\right\}, \\
& i=1 \ldots N
\end{aligned}
$$

The resulting full conditional distribution for the approximated functions $\mathbf{w}_{i}$ is such that

$$
\begin{aligned}
& f\left(\mathbf{w}_{i} \mid \text { rest }\right) \propto \\
& \quad \exp \left[-\frac{1}{2}\left(\left(\mathbf{X}_{i}\left(\mathbf{h}_{i}\right)-\left(z_{0 i} \mathbf{1}+z_{1 i} \mathbf{f}\right)\right)^{\prime} \gamma_{R} \boldsymbol{\Sigma}^{-1}\left(\mathbf{X}_{i}\left(\mathbf{h}_{i}\right)-\left(z_{0 i} \mathbf{1}+z_{1 i} \mathbf{f}\right)\right)\right)\right] * \\
& \quad \exp \left[-\frac{1}{2}\left(\mathbf{w}_{i}^{\prime}\left(\gamma_{w}^{-1} \mathbf{\Sigma}+\lambda_{w}^{-1} \mathbf{P}_{w}\right)^{-1} \mathbf{w}_{i}\right)\right] \mathbf{1}\left\{t_{1}+\sum_{k=2}^{p}\left(t_{k}-t_{k-1}\right) \exp \left(w_{i}\left(t_{k-1}\right)\right)=t_{p}\right\} .
\end{aligned}
$$

This full conditional does not have a known distributional form and can be sampled from via a Metropolis step in a MCMC sampler.

The components of the joint distribution of the data and all unknown parameters that are dependent on $\mathbf{X}_{i}$ are the sampling distribution (5) and equation (8). The resulting full conditional distribution is such that

$$
\begin{aligned}
& f\left(\mathbf{X}_{i} \mid \text { rest }\right) \propto \\
& \exp \left[-\frac{1}{2 \sigma_{Y}^{2}}\left(\mathbf{Y}_{i}-\mathbf{X}_{i}\right)^{\prime}\left(\mathbf{Y}_{i}-\mathbf{X}_{i}\right)\right] * \\
& \exp \left[-\frac{1}{2}\left(\left(\mathbf{X}_{i}\left(\mathbf{h}_{i}\right)-\left(z_{0 i} \mathbf{1}+z_{1 i} \mathbf{f}\right)\right)^{\prime} \boldsymbol{\Sigma}_{X}^{-1}\left(\mathbf{X}_{i}\left(\mathbf{h}_{i}\right)-\left(z_{0 i} \mathbf{1}+z_{1 i} \mathbf{f}\right)\right)\right)\right] .
\end{aligned}
$$

This full conditional distribution also is not of a known distributional form and can be sampled from using a Metropolis step. However, as significant features of the 
unregistered function, $\mathbf{X}_{i}$, should be unchanged by the registration. Smoothness in the registered function, $\mathbf{X}_{i}\left(\mathbf{h}_{i}\right)$, implies the same level of smoothness in the unregistered function $\mathbf{X}_{i}$. For ease of sampling, we will re-write (8) in terms of the unregistered function, $\mathbf{X}_{i}$ so that

$$
\begin{aligned}
& f\left(\mathbf{X}_{i} \mid\right.\text { rest }) \propto \\
& \exp \left[-\frac{1}{2 \sigma_{Y}^{2}}\left(\mathbf{Y}_{i}-\mathbf{X}_{i}\right)^{\prime}\left(\mathbf{Y}_{i}-\mathbf{X}_{i}\right)\right] * \\
& \exp \left[-\frac{1}{2}\left(\left(\mathbf{X}_{i}-\left(z_{0 i} \mathbf{1}+z_{1 i} \mathbf{f}\left(\mathbf{h}_{i}^{-1}\right)\right)\right)^{\prime} \boldsymbol{\Sigma}_{X}^{-1}\left(\mathbf{X}_{i}-\left(z_{0 i} \mathbf{1}+z_{1 i} \mathbf{f}\left(\mathbf{h}_{i}^{-1}\right)\right)\right)\right)\right]
\end{aligned}
$$

which results in a multivariate normal full conditional distribution for $\mathbf{X}_{i}$.

When noisy observations, $\mathbf{Y}_{i}, i=1, \ldots, N$ are recorded, the approximation we make in (10), while preserving conjugacy, prevents exact variational Bayes updates to be performed on the approximate posterior distributions for the following parameters: $\mathbf{X}_{i}$, $i=1 \ldots N, \sigma_{Y}^{2}, \eta_{X}$, and $\lambda_{X}$. Hence, the adapted variational Bayes procedure proposed here requires special handling under this data assumption.

\section{C.2 Adapted Variational Bayes For Noisy Functional Data}

In the traditional variational Bayes and AVB algorithms, it is assumed that $E_{\left(\boldsymbol{\theta}_{-k}\right)}\left(\log f\left(\boldsymbol{\theta}_{k} \mid\right.\right.$ rest $)$ can be evaluated for all parameters with conditionally conjugate prior distributions. For the registration model that accounts for noisy observations, some of these expectations cannot be determined exactly. However, we can adjust the original AVB algorithm further to perform an approximate inference procedure under these conditions. With these adjustments, the convergence properties of the adapted variational Bayes algorithm no longer hold. Nevertheless, we have found in practice that the adjusted algorithm still results in useful estimates for initializing a MCMC sampler.

Here we look at why the approximate posterior distributions for $\mathbf{X}_{i}, i=1, \ldots, N$, $\eta_{X}$, and $\lambda_{X}$ cannot be updated properly using the adapted variational Bayes algorithm. In the $m^{t h}$ iteration, the following update should be made to $\log q\left(\mathbf{X}_{i}\right)$, for $i=1 \ldots N$ :

$$
\log \left[q^{(m)}\left(\mathbf{X}_{i}\right)\right] \propto E_{\left(\theta_{-\mathbf{x}_{i}}\right)}\left[\log f\left(\mathbf{X}_{i} \mid r e s t\right)\right]
$$

where

$$
\begin{aligned}
E_{\left(\theta-\mathbf{x}_{i}\right)}\left[\log f\left(\mathbf{X}_{i} \mid \text { rest }\right)\right] & \propto E_{\left(\theta_{-} \mathbf{x}_{i}\right)}\left[-\frac{1}{2}\left[\left(\mathbf{X}_{i}-\boldsymbol{\mu}_{\mathbf{X}_{i} \mid r e s t}\right)^{\prime} \mathbf{\Sigma}_{\mathbf{X}_{i} \mid r e s t}^{-1}\left(\mathbf{X}_{i}-\boldsymbol{\mu}_{\mathbf{X}_{i} \mid \text { rest }}\right)\right]\right] \\
\boldsymbol{\Sigma}_{\mathbf{X}_{i} \mid \text { rest }} & =\left(\frac{1}{\sigma_{Y}^{2}} \mathbf{I}_{p}+\eta_{X} \mathbf{P}_{1}^{-}+\lambda_{X} \mathbf{P}_{2}^{-}\right)^{-1}, \text { and } \\
\boldsymbol{\mu}_{\mathbf{X}_{i} \mid \text { rest }} & =\boldsymbol{\Sigma}_{\mathbf{X}_{i} \mid \text { rest }}\left[\frac{1}{\sigma_{Y}^{2}} \mathbf{Y}_{i}+\left(\eta_{X} \mathbf{P}_{1}^{-}+\lambda_{X} \mathbf{P}_{2}^{-}\right)\left(z_{0 i} \mathbf{1}_{p}+z_{1 i} \mathbf{f}\left(\mathbf{h}_{i}^{-1}\right)\right)\right] .
\end{aligned}
$$


Taking the expectation over the $q$ distributions for all other parameters except for the base functions results to the following updated parameters of $q\left(\mathbf{X}_{i}\right)=N_{p}\left(\boldsymbol{\mu}_{q\left(\mathbf{X}_{i}\right)}, \boldsymbol{\Sigma}_{q\left(\mathbf{X}_{i}\right)}\right)$,

$\boldsymbol{\Sigma}_{q\left(\mathbf{X}_{i}\right)}^{(m)}=\left(\mu_{q\left(\frac{1}{\sigma_{Y}^{2}}\right)} \mathbf{I}_{p}+\mu_{q\left(\eta_{X}\right)} \mathbf{P}_{1}^{-}+\mu_{q\left(\lambda_{X}\right)} \mathbf{P}_{2}^{-}\right)^{-1}$, and

$\boldsymbol{\mu}_{q\left(\mathbf{X}_{i}\right)}^{(m)}=\boldsymbol{\Sigma}_{q\left(\mathbf{X}_{i}\right)}^{(m)}\left[\mu_{q\left(\frac{1}{\sigma_{Y}^{2}}\right)} \mathbf{Y}_{i}+\left(\mu_{q\left(\eta_{X}\right)} \mathbf{P}_{1}^{-}+\mu_{q\left(\lambda_{X}\right)} \mathbf{P}_{2}^{-}\right)\left(\mu_{q\left(z_{0 i}\right)} \mathbf{1}_{p}+\mu_{q\left(z_{1 i}\right)} E_{\left(\theta_{-\mathbf{x}_{i}}\right)}\left[\mathbf{f}\left(\mathbf{h}_{i}^{-1}\right)\right]\right)\right]$

In (11), the expectation of $\mathbf{f}\left(\mathbf{h}_{i}^{-1}\right)$ is unknown. So, the first approximation we will make is that $E_{\left(\theta_{\left.-\mathbf{x}_{i}\right)}\right.}\left[\mathbf{f}\left(\mathbf{h}_{i}^{-1}\right)\right] \approx \boldsymbol{\mu}_{q(\mathbf{f})}\left(\mathbf{h}_{i}^{-1}\right)$.

Similarly, to update $\log q\left(\eta_{X}\right)$ :

$$
\log \left[q^{(m)}\left(\eta_{X}\right)\right] \propto E_{\left(\theta_{-\eta_{X}}\right)}\left[\log f\left(\eta_{X} \mid \text { rest }\right)\right]
$$

where

$$
\begin{aligned}
E_{\left(\theta_{\left.-\eta_{X}\right)}\right.}\left[\log f\left(\eta_{X} \mid \text { rest }\right)\right] & \propto E_{\left(\theta_{\left.-\eta_{X}\right)}\left[c_{\eta_{X} \mid \text { rest }} \log \eta_{X}-d_{\eta_{X} \mid \text { rest }} \eta_{X}\right]\right.} \\
c_{\eta_{X} \mid \text { rest }} & =N+c, \text { and } \\
d_{\eta_{X} \mid \text { rest }} & =d+\frac{1}{2} \sum_{i=1}^{N} \operatorname{tr}\left[\left(\mathbf{X}_{i}-\left(z_{0 i} \mathbf{1}_{p}+z_{1 i} \mathbf{f}\left(\mathbf{h}_{i}^{-1}\right)\right)\right)\left(\mathbf{X}_{i}-\left(z_{0 i} \mathbf{1}_{p}+z_{1 i} \mathbf{f}\left(\mathbf{h}_{i}^{-1}\right)\right)\right)^{\prime} \mathbf{P}_{1}^{-}\right] .
\end{aligned}
$$

Taking the expectation over the $q$ distributions for all other parameters except for the base functions results to the following updated parameters of $q\left(\eta_{X}\right)=G\left(c_{q\left(\eta_{X}\right)}, d_{q\left(\eta_{X}\right)}\right)$,

$$
\begin{aligned}
c_{q\left(\eta_{X}\right)}^{(m)}= & N+c \\
d_{q\left(\eta_{X}\right)}^{(m)}= & d+\frac{1}{2} \operatorname{tr}\left[\left(\sum _ { i = 1 } ^ { N } \left(\boldsymbol{\Sigma}_{q\left(\mathbf{X}_{i}\right)}+\boldsymbol{\mu}_{q\left(\mathbf{X}_{i}\right)} \boldsymbol{\mu}_{q\left(\mathbf{X}_{i}\right)}^{\prime}-2 \boldsymbol{\mu}_{q\left(\mathbf{X}_{i}\right)}\left(\mu_{q\left(z_{0 i}\right)} \mathbf{1}_{p}+\mu_{q\left(z_{1 i}\right)} E_{\left(\theta_{-\eta_{X}}\right)}\left[\mathbf{f}\left(\mathbf{h}_{i}^{-1}\right)\right]\right)^{\prime}\right.\right.\right. \\
& \left.+2 \mu_{q\left(z_{0 i}\right)} \mu_{q\left(z_{1 i}\right)} \mathbf{1}_{p} E_{\left(\theta_{-\eta_{X}}\right)}\left[\mathbf{f}\left(\mathbf{h}_{i}^{-1}\right)\right]^{\prime}+\left(\sigma_{q\left(z_{1 i}\right)}^{2}+\mu_{q\left(z_{1 i}\right)}^{2}\right) E_{\left(\theta_{-\eta_{X}}\right)}\left[\mathbf{f}\left(\mathbf{h}_{i}^{-1}\right) \mathbf{f}\left(\mathbf{h}_{i}^{-1}\right)^{\prime}\right]\right), \text { and } \\
& \left.\left.+\left(2 \sum_{i=1}^{N-1}\left(\sigma_{q\left(z_{0 i}\right)}^{2}+\mu_{q\left(z_{0 i}\right)}^{2}\right)+\sum_{i=1}^{N-1} \sum_{j=1}^{N-1} \mu_{q\left(z_{0 i}\right)} \mu_{q\left(z_{0 j}\right)} \mathbf{1}\{j \neq i\}\right) \mathbf{1}_{p} \mathbf{1}_{p}^{\prime}\right) \mathbf{P}_{1}^{-}\right] .
\end{aligned}
$$

In the expression for $d_{q\left(\eta_{X}\right)}^{(m)}, E_{\left(\theta_{-\eta_{X}}\right)}\left[\mathbf{f}\left(\mathbf{h}_{i}^{-1}\right)\right]$ and $E_{\left(\theta_{-\eta_{X}}\right)}\left[\mathbf{f}\left(\mathbf{h}_{i}^{-1}\right) \mathbf{f}\left(\mathbf{h}_{i}^{-1}\right)^{\prime}\right]$ are unknown. Thus, we will make the following approximations,

$$
E_{\left(\theta_{-\eta_{X}}\right)}\left[\mathbf{f}\left(\mathbf{h}_{i}^{-1}\right)\right] \approx \boldsymbol{\mu}_{q(\mathbf{f})}\left(\mathbf{h}_{i}^{-1}\right) \text { and } E_{\left(\theta_{-\eta_{X}}\right)}\left[\mathbf{f}\left(\mathbf{h}_{i}^{-1}\right) \mathbf{f}\left(\mathbf{h}_{i}^{-1}\right)^{\prime}\right] \approx \boldsymbol{\Sigma}_{q\left(\mathbf{X}_{i}\right)} / N+\boldsymbol{\mu}_{q(\mathbf{f})}\left(\mathbf{h}_{i}^{-1}\right) \boldsymbol{\mu}_{q(\mathbf{f})}\left(\mathbf{h}_{i}^{-1}\right)^{\prime} .
$$

Note, $\boldsymbol{\Sigma}_{q\left(\mathbf{X}_{i}\right)}$ does not depend on $i$.

The variational Bayes algorithm update for $\lambda_{X}$ is similar to that of $\eta_{X}$ and requires the same approximations,

$$
\log \left[q^{(m)}\left(\lambda_{X}\right)\right] \propto E_{\left(\theta_{-\lambda_{X}}\right)}\left[\log f\left(\lambda_{X} \mid r e s t\right)\right]
$$


where

$$
\begin{aligned}
E_{\left(\theta_{\left.-\lambda_{X}\right)}\right.}\left[\log f\left(\lambda_{X} \mid \text { rest }\right)\right] & \propto E_{\left(\theta_{-\lambda_{X}}\right)}\left[c_{\lambda_{X} \mid \text { rest }} \log \lambda_{X}-d_{\lambda_{X} \mid \text { rest }} \lambda_{X}\right] \\
c_{\lambda_{X} \mid \text { rest }} & =N\left(\frac{p-2}{2}\right)+c, \text { and } \\
d_{\eta_{X} \mid \text { rest }} & =d+\frac{1}{2} \sum_{i=1}^{N} \operatorname{tr}\left[\left(\mathbf{X}_{i}-\left(z_{0 i} \mathbf{1}_{p}+z_{1 i} \mathbf{f}\left(\mathbf{h}_{i}^{-1}\right)\right)\right)\left(\mathbf{X}_{i}-\left(z_{0 i} \mathbf{1}_{p}+z_{1 i} \mathbf{f}\left(\mathbf{h}_{i}^{-1}\right)\right)\right)^{\prime} \mathbf{P}_{2}^{-}\right] .
\end{aligned}
$$

Taking the expectation over the $q$ distributions for all other parameters except for the base functions results to the following updated parameters of $q\left(\lambda_{X}\right)=G\left(c_{q\left(\lambda_{X}\right)}, d_{q\left(\lambda_{X}\right)}\right)$,

$$
\begin{aligned}
c_{q\left(\lambda_{X}\right)}^{(m)}= & N\left(\frac{p-2}{2}\right)+c, \text { and } \\
d_{q\left(\lambda_{X}\right)}^{(m)}= & d+\frac{1}{2} \operatorname{tr}\left[\left(\sum _ { i = 1 } ^ { N } \left(\boldsymbol{\Sigma}_{q\left(\mathbf{X}_{i}\right)}+\boldsymbol{\mu}_{q\left(\mathbf{X}_{i}\right)} \boldsymbol{\mu}_{q\left(\mathbf{X}_{i}\right)}^{\prime}-2 \boldsymbol{\mu}_{q\left(\mathbf{X}_{i}\right)}\left(\mu_{q\left(z_{0 i}\right)} \mathbf{1}_{p}+\mu_{q\left(z_{1 i}\right)} E_{\left(\theta_{-\lambda_{X}}\right)}\left[\mathbf{f}\left(\mathbf{h}_{i}^{-1}\right)\right]\right)^{\prime}\right.\right.\right. \\
& \left.+2 \mu_{q\left(z_{0 i}\right)} \mu_{q\left(z_{1 i}\right)} \mathbf{1}_{p} E_{\left(\theta_{-\lambda_{X}}\right)}\left[\mathbf{f}\left(\mathbf{h}_{i}^{-1}\right)\right]^{\prime}+\left(\sigma_{q\left(z_{1 i}\right)}^{2}+\mu_{q\left(z_{1 i}\right)}^{2}\right) E_{\left(\theta_{\left.-\lambda_{X}\right)}\right.}\left[\mathbf{f}\left(\mathbf{h}_{i}^{-1}\right) \mathbf{f}\left(\mathbf{h}_{i}^{-1}\right)^{\prime}\right]\right) \\
& \left.\left.+\left(2 \sum_{i=1}^{N-1}\left(\sigma_{q\left(z_{0 i}\right)}^{2}+\mu_{q\left(z_{0 i}\right)}^{2}\right)+\sum_{i=1}^{N-1} \sum_{j=1}^{N-1} \mu_{q\left(z_{0 i}\right)} \mu_{q\left(z_{0 j}\right)} \mathbf{1}\{j \neq i\}\right) \mathbf{1}_{p} \mathbf{1}_{p}^{\prime}\right) \mathbf{P}_{2}^{-}\right]
\end{aligned}
$$

Again, in the expression for $d_{q\left(\lambda_{X}\right)}^{(m)}, E_{\left(\theta_{-\lambda_{X}}\right)}\left[\mathbf{f}\left(\mathbf{h}_{i}^{-1}\right)\right]$ and $E_{\left(\theta_{-\lambda_{X}}\right)}\left[\mathbf{f}\left(\mathbf{h}_{i}^{-1}\right) \mathbf{f}\left(\mathbf{h}_{i}^{-1}\right)^{\prime}\right]$ are unknown. Thus, we will make the following approximations,

$$
E_{\left(\theta_{-\lambda_{X}}\right)}\left[\mathbf{f}\left(\mathbf{h}_{i}^{-1}\right)\right] \approx \boldsymbol{\mu}_{q(\mathbf{f})}\left(\mathbf{h}_{i}^{-1}\right) \text { and } E_{\left(\theta_{-\lambda_{X}}\right)}\left[\mathbf{f}\left(\mathbf{h}_{i}^{-1}\right) \mathbf{f}\left(\mathbf{h}_{i}^{-1}\right)^{\prime}\right] \approx \boldsymbol{\Sigma}_{q\left(\mathbf{X}_{i}\right)} / N+\boldsymbol{\mu}_{q(\mathbf{f})}\left(\mathbf{h}_{i}^{-1}\right) \boldsymbol{\mu}_{q(\mathbf{f})}\left(\mathbf{h}_{i}^{-1}\right)^{\prime} .
$$

Due to these modifications, if noisy observations are observed the convergence properties of the adapted variational Bayes algorithm are not guaranteed to hold, and $\log [f(\mathbf{Y}, \mathbf{w} ; q)]$ cannot be monitored. However, we can monitor convergence for this model as follows. Taking advantage of the fact that functional smoothing converges more quickly than functional registration, fix the approximated unregistered functions, $\mathbf{X}_{i}, i=1, \ldots, N$, after a small number of iterations and proceed as if they are known. Then, as in the model where the observations are recorded without noise, $\log [f(\mathbf{X}, \mathbf{w} ; q)]$ can be monitored.

\section{C.3 The Berkeley Growth Data}

We refer back to the Berkeley Boys Growth Velocity dataset from Section 4.1. In Section 4.1, these data were smoothed prior to registration. Here, we again consider these functions with the added assumption that they are corrupted by simulated mean zero iid Gaussian noise, where the true noise variance, $\sigma_{Y}^{2}$, is .25.

While it is common in statistical analysis to perform preprocessing steps before applying a particular inference procedure, failing to account for the variability in parameter 

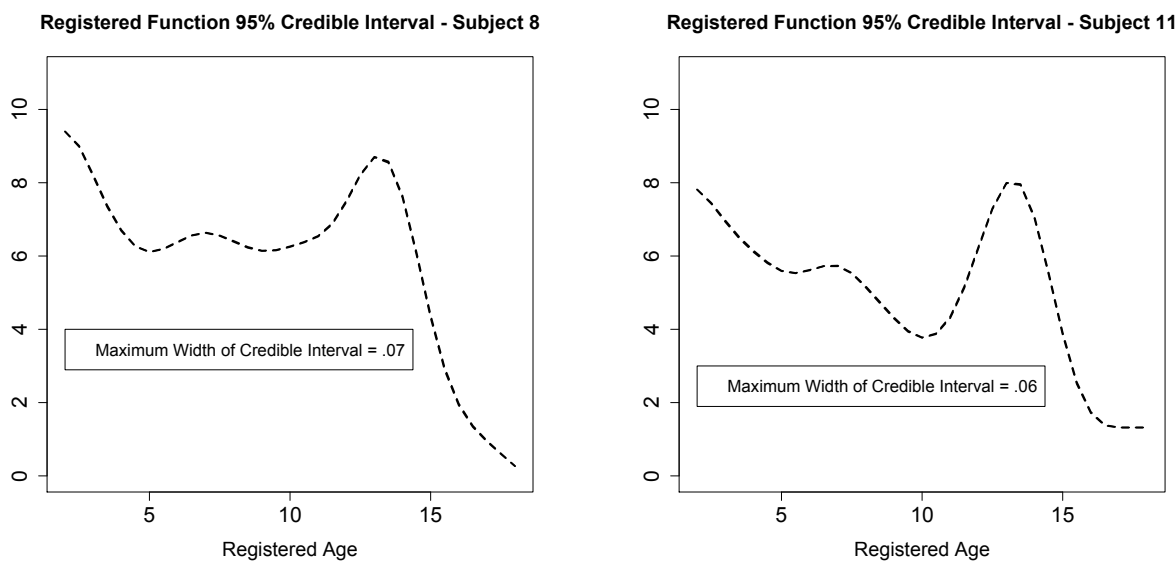

Figure C.1: Examples of Credible Intervals for Noiseless Observations . These are two examples from the Boys Growth Velocity Data of the tight credible bands that result from registering functions that are pre-smoothed. In Figure C.2, the top and lower right illustrations contain the credible intervals for these same observations when the noise process is included in the model.

estimates due to the preprocessing step leads to overly narrow confidence (or credible) regions. In some cases, the effect may be fairly small, and not much is lost in this oversight. However, as we show here, the underestimated variability can be substantial when uncertainty in the preprocessing steps is ignored.

In Section 4.2 is an illustration of how closely AVB and MCMC estimates of the registered functions adhere to one another. Not only do these estimates tend to be fairly similar when the functions are recorded without noise, but the uncertainty in these estimates is minimal. Figure C. 1 contains the credible bands for two of the 39 pre-smoothed Boys Growth Velocity Functions. These bands are so narrow the width between them cannot be seen. Keep in mind the posterior distributions of the registered functions are certainly multi-modal. These credible bands result from imposing the restriction that the mean value of the warping functions at each time point over the sample must equal that time point. Even with this restriction, the posterior distributions can be multi-modal. However, these narrow credible bands reflect that our estimates are in a highly probable area of the posterior distribution with minimal local variance. Figure C.2 contains credible bands for both the unregistered and registered functions for the same two functions used in Figure C.1 after noise has been added to the data and accounted for in the model. The variability due to noise is substantial. The solid line in all of the plots contains the noiseless version of these estimates (or observations in the case of the unregistered functions).

In addition to providing more accurate credible intervals, this model estimates the noise variance to be .258 (actual noise variance is .25). This estimate is obtained using uninformative priors for both the noise variance, $\sigma_{Y}^{2}$ and the associated smoothing 

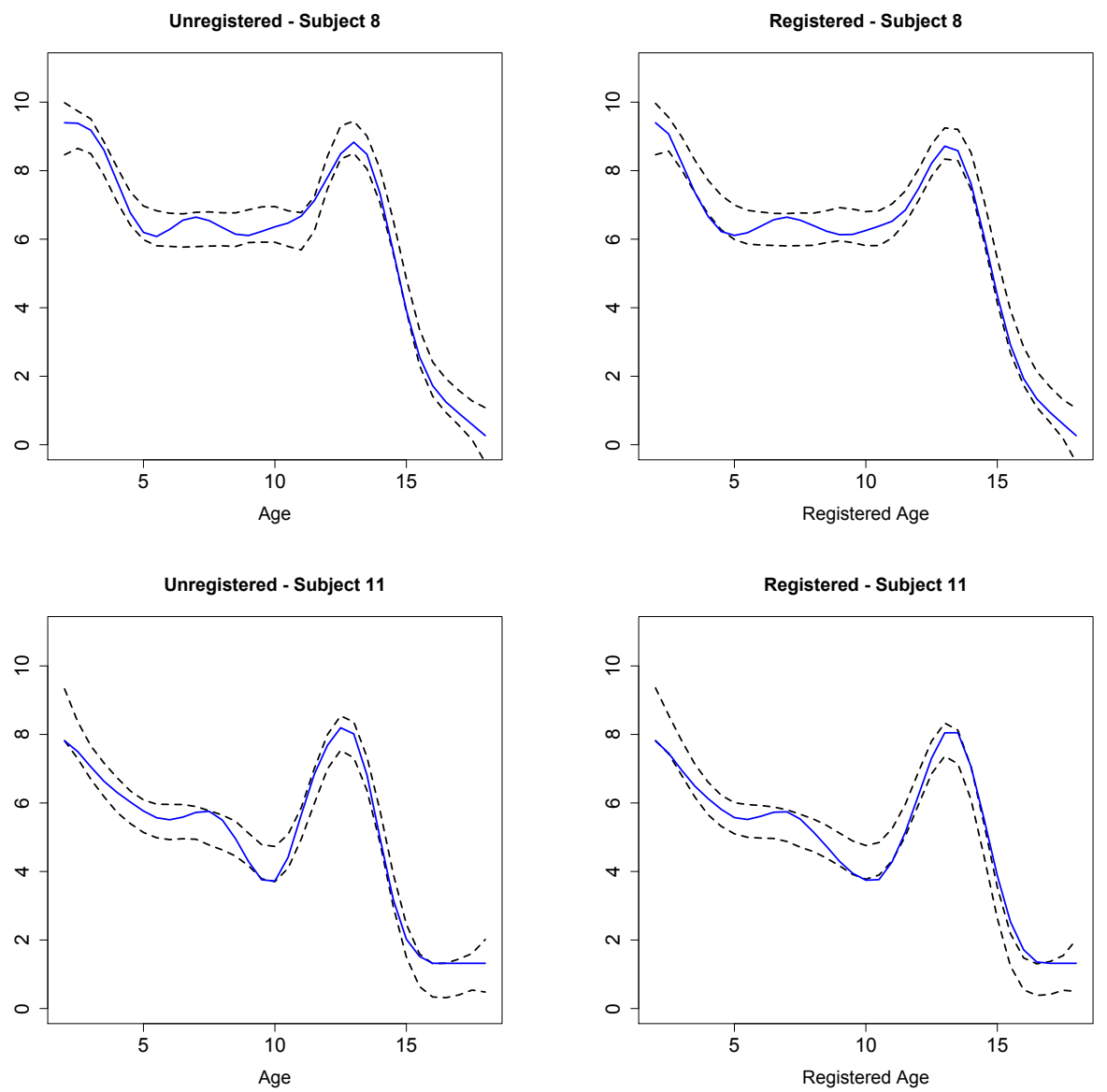

Figure C.2: Examples of Credible Bands for the Unregistered and Registered Functions when the Noise Process is Included in the Model. Top and Lower Left 95\% credible bands for the unregistered functions are plotted with the original noiseless functions for subjects 8 and 11. Top and Lower Right For subjects 8 and 11, 95\% credible bands for the registered functions are plotted with the estimate of the registered 'true' functions. 
parameter $\lambda_{X}$.

This analysis illustrates how regularizing the data prior to statistical analysis for registration models severely limits inference for these models. If significant noise is present in the data it is prudent to account for the variability in the registration process due to the noise. Our proposed hierarchical model is one way to account for this variability.

\section{References}

Earls, C. and Hooker, G. (2014). "Bayesian covariance estimation and inference in latent Gaussian process models." Statistical Methodology, 18: 79-100. 3

Raket, L., Sommer, S., and Markussen, B. (2014). "A nonlinear mixed-effects model for simultaneous smoothing and registration of functional data." Pattern Recognition Letters, 38: 1-7. 10 